\title{
Suppression of Dynamically Induced Stochastic Magnetic Behaviour through Materials Engineering
}

\author{
T.J. Broomhall ${ }^{1}$, A.W. Rushforth ${ }^{2}$, M.C. Rosamond ${ }^{3}$, E.H. Linfield ${ }^{3}$ and T.J. Hayward ${ }^{1}$ \\ ${ }^{1}$ Department of Materials Science and Engineering, University of Sheffield, Sheffield, UK \\ ${ }^{2}$ School of Physics and Astronomy, University of Nottingham, Nottingham, NG7 2RD UK \\ ${ }^{3}$ School of Electronic and Electrical Engineering, University of Leeds, Leeds, UK
}

\begin{abstract}
Stochastic behaviour fundamentally limits the performance and reliability of nanomagnetic devices. Typically, stochastic behaviour is assumed to be the result of simple thermal activation, but it may also be "dynamically induced" i.e. a direct result of the spatial and temporal complexity of magnetisation dynamics. In this paper, we show how materials engineering can be used to comprehensively suppress dynamically induced stochasticity. Using the dynamics of magnetic domain walls in $\mathrm{Ni}_{80} \mathrm{Fe}_{20}$ nanowires as a case study we show how manipulation of the Gilbert damping constant via doping with the rare earth element Terbium dramatically simplifies domain wall dynamics. This allows us to obtain quasi-deterministic behaviours from systems that nominally exhibit exceptionally high levels of stochasticity.
\end{abstract}

\section{Introduction}

Overcoming stochastic behaviour is one of the great challenges of developing information storage and processing technologies based on nanopatterned magnetic materials. For example, the areal density (numbers of bits stored per inch ${ }^{2}$ ) in conventional magnetic hard disk drives is fundamentally limited by the "trilemma" of magnetic data storage,${ }^{1}$ the key consideration of which is preventing the random reversal of data bits. Similar considerations must also be made in the design of solid-state magnetic random access memory (MRAM) cells, where stochastic effects can cause both data loss, and result in write errors when the states of the devices are intentionally addressed. ${ }^{2}$

Stochastic effects in magnetic devices are typically assumed to be caused by thermal activation, as described by Néel-Brown theory. ${ }^{3}$ These can be suppressed by careful materials engineering, for example by strengthening a material's magnetocrystalline anisotropy to increase the energy barrier between magnetisation states. However, stochastic behaviour can also arise from the inherent spatial and temporal complexity of magnetisation dynamics. ${ }^{4-10} \mathrm{~A}$ notable example of this occurs in the propagation of magnetic domain walls (DWs) in nanowires composed of the soft ferromagnetic material $\mathrm{Ni}_{80} \mathrm{Fe}_{20} .{ }^{11-17}$ These were studied extensively in early efforts to create racetrack memory devices $^{18,19}$ and remain relevant to a diverse range of device applications ${ }^{20-30}$ and studies of fundamental magnetic behaviours. 5,31,32 
When propagated by magnetic fields ${ }^{33}$ or electric currents ${ }^{34}$ above a critical threshold, DWs undergo a process known as Walker breakdown, ${ }^{35}$ where their internal magnetisation structures undergo complex and continuous cycles of transformation. ${ }^{36,37}$ These transformations cause variable interactions with defect sites and result in both stochastic DW pinning, where inhibition of DW propagation by a defect becomes probabilistic, ${ }^{12,14,17,38,39}$ and stochastic DW depinning, where the distribution of fields/currents required to depin DWs from defects adopt multi-mode characters. ${ }^{11,13,15,16,38,39}$. We refer to these technologically inhibitive phenomena as "dynamically induced" stochastic effects to differentiate them from their simpler, thermally activated counterparts. While propagating DWs at fields/currents below Walker breakdown offers a route to supressing dynamically induced stochasticity ${ }^{14,15}$, Walker Breakdown fields/currents typically lie below those required to initiate DW propagation (for example due to pinning by edge roughness), meaning that no regime is available where DWs can be propagated without exhibiting stochastic behaviours.

In this paper, we demonstrate that materials engineering can be used to comprehensively suppress dynamically induced stochastic effects in DW devices. We show that by manipulating the nanowires' Gilbert damping constants via doping with the rare earth element Terbium, we can push the onset of complex Walker breakdown dynamics outside of the devices' normal operating windows (i.e. the applied fields required to inject and propagate DWs). This greatly simplifies the dynamics of the DWs such that they exhibit consistent and repeatable interactions with defect sites. Thus, by addressing the root cause of the DWs' stochastic behaviours we obtain simple quasi-deterministic switching behaviours from devices that are nominally highly stochastic. In doing so we provide a powerful illustration of how materials engineering can be used to produce inherently reliable device behaviour even in systems where the origin of stochasticity lies beyond simple thermal activation.

\section{Methods}

To examine the effect of the Gilbert damping constant on the stochasticity of DW pinning we first deposited both undoped and $5 \% \mathrm{~Tb}$-doped $\mathrm{Ni}_{80} \mathrm{Fe}_{20}$ precursor thin films by co-sputtering from $\mathrm{Ni}_{80} \mathrm{Fe}_{20}$ and Tb targets onto naturally oxidised Si substrates. All films had a thickness of $20 \mathrm{~nm}$ and were capped by a $2 \mathrm{~nm}$ thick layer of Ru to prevent oxidation. The precursor films were then fabricated into $400 \mathrm{~nm}$ wide nanowires via ion milling through a thermally evaporated $\mathrm{Al} / \mathrm{Ti}$ hard mask, patterned by electron beam lithography and lift-off processing

The thin films' field-angle resolved hysteresis loops were measured using a home-built MOKE magnetometer (spot size several 100s of $\mu \mathrm{m}$ ) with a maximum field amplitude of $\mathrm{H}= \pm 300$ Oe. These allowed extraction of films' easy axis coercivities, $H_{c}$, and anisotropy fields, $H_{k}$. The films' Gilbert damping constants, $\alpha$, and saturation magnetisations, $M_{s}$, were measured by field-swept FMR 
measurements using a vector network analyser ferromagnetic resonance (VNA-FMR) system operating in a 1-port (reflection) mode. Measurements typically produced mixing of the real and imaginary parts of the dynamic susceptibility, which we corrected for in our fitting using the approach of Kalarickal. ${ }^{40}$ Values of $M_{s}$ were extracted by fits to the Kittel equation: ${ }^{40}$

$$
f_{\text {res }}=\frac{\mu_{0} \gamma}{2 \pi} \sqrt{\left(H+H_{K}\right)\left(H+H_{K}+M_{S}\right)} \quad \text { (Equation 1) }
$$

Where $f_{\text {res }}$ was films' FMR frequency at applied field $H, H_{K}$ was the films anisotropy field, $\gamma$ was the gyromagnetic ratio and $\mu_{0}$ was the permeability of free space Values of $\alpha$ were extracted from the variation of the field-swept FMR linewidth, $\Delta H$, with excitation frequency, $f$, using ${ }^{40}$ :

$$
\Delta H=\Delta H_{0}+\frac{4 \pi \alpha f}{|\gamma| \mu_{0}} \quad \text { (Equation 2) }
$$

where $\Delta H_{0}$ were the films' extrinsic FMR line-widths.

Measurements of the magnetisation switching of the nanowires were performed using a home built focused magneto-optic Kerr effect magnetometer (FMOKE) with a laser spot size of $\sim 3 \mu \mathrm{m}$. Signal-tonoise levels were high enough to allow measurement of individual switching events with a high-degree of fidelity.

Figure 1(a) illustrates the geometry of the nanowire devices we used characterised in this study. The devices consisted of $400 \mathrm{~nm}$ wide, $20 \mathrm{~nm}$ thick $\mathrm{Ni}_{80} \mathrm{Fe}_{20}$ nanowires connected to $10 \times 10 \mu \mathrm{m}^{2}$ nucleation pads, which were used to inject DWs into the nanowires. Nanowires of this geometry were expected to favour vortex domain wall (VDW) configurations with either clockwise (CW) or anticlockwise (ACW) chirality. ${ }^{41-43}$ Lithographically patterned notches, placed at the mid-point of the nanowires, were used as artificial defect sites at which to probe the stochasticity of DW pinning. The defect sites were patterned in symmetrical "double notch" configurations to ensure that our results were not distorted by VDWs exhibiting chirality dependent interactions with the defect sites as would occur for asymmetric, single notch designs. ${ }^{11}$ To characterise the pinning of the DWs at the notches we performed focused magneto-optic Kerr effect (FMOKE) measurements at locations both before and after the defect sites in each nanowire studied (Figure 1(a)). A sinusoidal applied field with amplitude +/- 300 Oe was continuously cycled at a rate $27 \mathrm{~Hz}$ while 100 single-shot hysteresis loops were obtained at each location. These allowed us to reconstruct the DWs' injection field distributions (IFDs, applied fields required to move the DW from the pad to the defect site) and depinning field distributions (DFDs, fields required to move the DW from the notch to the end of the nanowire).

Our understanding of the experimental measurements was supported by micromagnetic simulations of DW dynamics, performed using the GPU accelerated Mumax ${ }^{3}$ simulation package. ${ }^{44}$ Simulations 
used meshes of either $2.5 \times 2.5 \times 2.5 \mathrm{~nm}^{3}$ or $2 \times 2 \times 2 \mathrm{~nm}^{3}$ depending on which was required to achieve convergence. Moving simulation windows were used to keep the DW centred in the mesh during propagation in all simulations except those where the DWs' interactions with defect sites were explicitly modelled. Values of $M_{s}$ and $\alpha$ were taken from the results of the FMR measurements, while the exchange stiffness was given a standard value of $A_{e x}=13 \mathrm{pJm}^{-1}$. The films' weak magnetocrystalline anisotropies were neglected due to the dominance of shape anisotropy on the DWs' behaviours.

(a)
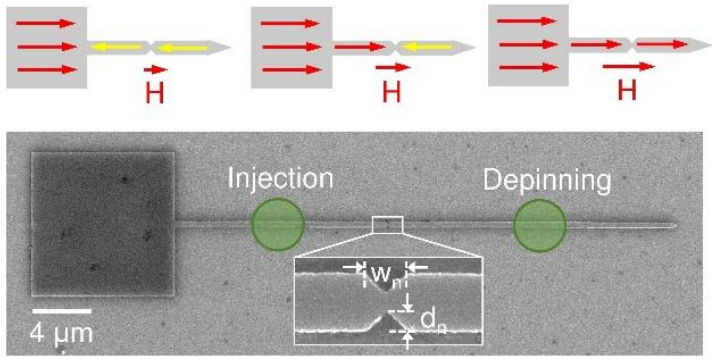

(b)

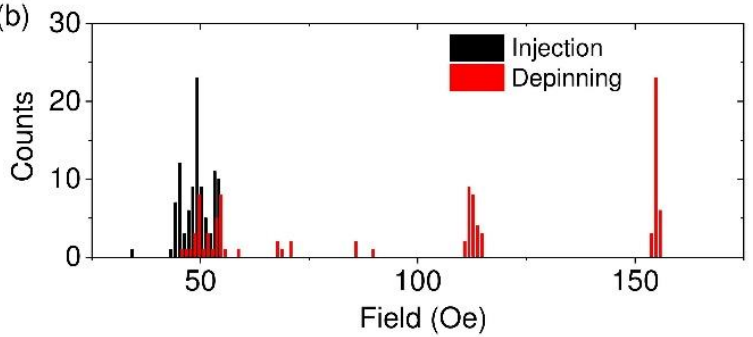

Figure 1: (a) SEM images illustrating the geometry of the nanowire devices studied. The green circles illustrate the approximate position of the FMOKE laser spot when characterising a devices' IFDs (Injection) and DFDs (depinning). The schematic at the top of the figure illustrates how the magnetisation configuration of the devices is expected to evolve as the applied field is increased. (b) Example IFD and DFD measured from a $\mathrm{Ni}_{80} \mathrm{Fe}_{20}$ nanowire device with $d_{n}=85 \mathrm{~nm}$ and $w_{n}=215 \mathrm{~nm}$.

\section{Results and Discussion}

We begin with an illustration of how stochastic DW pinning typically manifests in $\mathrm{Ni}_{80} \mathrm{Fe}_{20}$ nanowire devices. Figure 1 (b) presents typical IFDs and DFDs measured for a $\mathrm{Ni}_{80} \mathrm{Fe}_{20}$ nanowire containing notches with depths $d_{n}=85 \mathrm{~nm}$ and widths $w_{n}=215 \mathrm{~nm}$. While the IFD was relatively well-defined, consisting of a few tightly spaced peaks at $~ 50$ Oe, the DFD was highly stochastic and exhibited both key dynamically induced stochastic phenomena that are typically observed for such nanowire devices: ${ }^{38}$ Stochastic pinning can be observed in the partial overlapping of the IFD and DFD, indicating that a sizeable fraction of DWs passed through the defect site without stopping. Stochastic depinning can be observed in the multimode character of the DFD, with each mode representing a distinct DW configuration becoming pinned at the defect site..$^{4,7,39}$ 
To engineering magnetic materials that inherently suppressed dynamically induced stochastic pinning phenomena we adopted an approach we previously demonstrated in a micromagnetic modellingbased study: ${ }^{43}$ The Walker breakdown field $\left(\mathrm{H}_{\mathrm{WB}}\right)$, which represents the point of onset of dynamically induced stochastic effects, is predicted to increase in proportion to the Gilbert damping constant $\alpha$ in simple analytical models. ${ }^{43}$ Thus, by enhancing $\alpha$ it was expected to be possible to push $\mathrm{H}_{\mathrm{WB}}$ beyond a device's normal operating field/current range, stabilising DW motion, and thus suppressing stochasticity.

Doping $\mathrm{Ni}_{80} \mathrm{Fe}_{20}$ with rare earth elements ( $\mathrm{Tb}, \mathrm{Ho}, \mathrm{Dy}$ ) has been shown to increase the Gilbert damping constant from its nominal value of $\alpha=0.007$, with $\sim 5 \%$ doping being sufficient to produce enhancements of around an order of magnitude ${ }^{45-47}$. The enhancement of damping with rare earth concentration is explained by slow relaxing rare earth impurity model ${ }^{46}$. Nanowires fabricated from the $5 \% \mathrm{~Tb}$ doped $\mathrm{Ni}_{80} \mathrm{Fe}_{20}$ precursor films were expected to increase $\mathrm{H}_{\text {WB }}$ from typical values of $\sim 10$ Oe to close to $100 \mathrm{Oe}$, well outside the range of typical DW propagation fields ( $<50$ Oe).
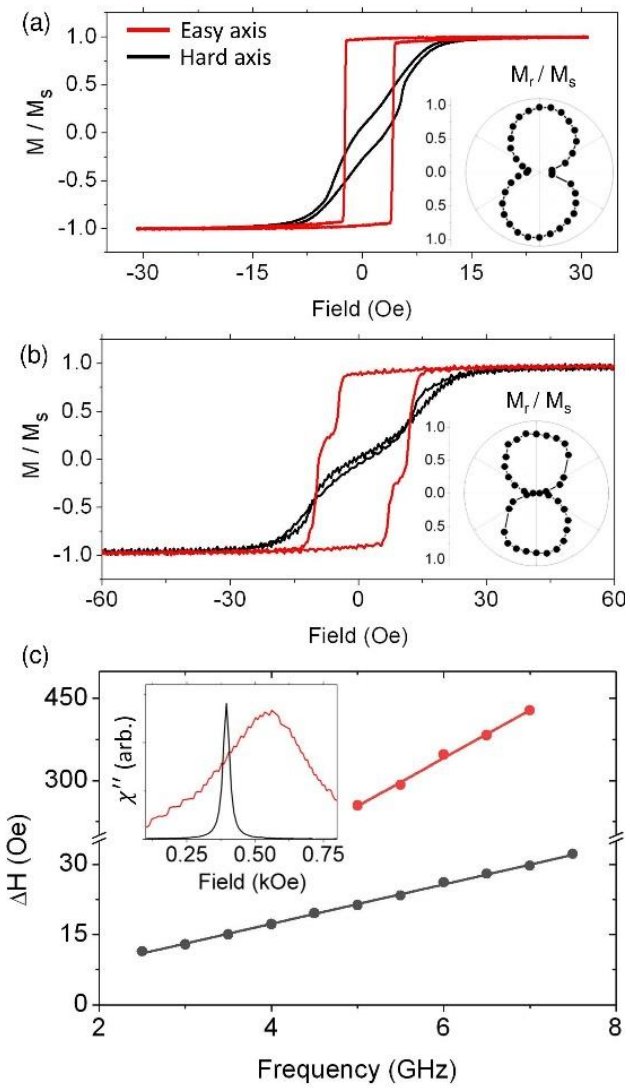

Figure 2: (a) Easy and hard axis hysteresis loops obtained from a $20 \mathrm{~nm}$ thick $\mathrm{Ni}_{80} \mathrm{Fe}_{20}$ thin film. The inset figure presents a polar plot of the film's remanence values. (b) Equivalent hysteresis loops and polar plots for a $20 \mathrm{~nm}$ thick $\mathrm{Ni}_{80} \mathrm{Fe}_{20}$ thin film doped with $5 \% \mathrm{~Tb}$. (c) Variation of the field-swept FMR linewidth with frequency for the undoped (black circles) and $5 \%$ Tb-doped (red circles) $\mathrm{Ni}_{80} \mathrm{Fe}_{20}$ thin film. The inset figure presents illustrative plots of the imaginary parts of the film's susceptibilities $\left(\chi^{\prime \prime}\right)$. Black curve: $\mathrm{Ni}_{80} \mathrm{Fe}_{20}$ film, $6 \mathrm{GHz}$ excitation. Red curve: $5 \% \mathrm{~Tb}$-doped $\mathrm{Ni}_{80} \mathrm{Fe}_{20}, 6.5 \mathrm{GHz}$ excitation. 
To establish that the $\mathrm{Tb}$-doped $\mathrm{Ni}_{80} \mathrm{Fe}_{20}$ films retained properties suitable for fabricating nanowire devices we characterised their magnetic properties using MOKE magnetometry, and compared them to those of undoped films (Figures $2(a) \&(b)$ ). In both cases the films exhibited weak uniaxial anisotropy and soft magnetic properties. The addition of Tb resulted in a modest enhancement of the easy axis coercivity from $H_{c}=3.30 \pm 0.05$ Oe to $H_{c}=10.0 \pm 0.5 \mathrm{Oe}$, and an increase in the anisotropy field (as estimated from the value of the films' saturation fields measured along their hard axes) from $\mathrm{H}_{\mathrm{K}}=18$ \pm 1 Oe to $H_{K}=61 \pm 3 \mathrm{Oe}$, with both these changes likely to have been due to the Tb-doping inducing a small increase in the film's magnetocrystalline anisotropy constants. ${ }^{45}$ We also noted a slight "doublestep" in the easy axis hysteresis loop of the doped film, and attributed this to competition between the shape anisotropy of the film's (approximately rectangular) substrate and the growth induced anisotropy of the film, which was misaligned by $\sim 30^{\circ}$ from the closest substrate edge. Critically, the coercivities of the Tb-doped films lay below typical injection fields for DW devices ( $\sim 50 \mathrm{Oe}^{4,39}$ ), and their anisotropy fields were substantially less than those that would be created by shape anisotropy upon patterning them into nanowires with a $400 \times 20 \mathrm{~nm}^{2}$ cross-section $\left(\sim 580 \mathrm{Oe}^{48}\right.$ ). We therefore concluded that the Tb-doped films were well-suited for patterning into nanowire devices.

Values of $\alpha$ for the Tb-doped and undoped films were measured using vector network analyser ferromagnetic resonance measurements (VNA-FMR). Figure 2(c) presents plots of the frequency dependence of the films' field swept line-widths, from which values of $\alpha$ were extracted by fits to Equation 1. The Tb-doped film was found to have $\alpha_{\mathrm{Tb} \text {-doped }}=0.120 \pm 0.005$, substantially higher than the value of $\alpha_{\text {undoped }}=0.0059 \pm 0.0001$ measured for the undoped film. This difference in $\alpha$ can be seen to manifest clearly in the differing gradients of the plots in figure 2(c), as well as in the widths of individual resonance peaks (see inset to figure 2(c)).

Fits of the field-dependence of the films' FMR resonance field frequency using Equation 2 allowed extraction of their saturation magnetisatiOons, with the Tb-doped film exhibiting a value of $M_{s}=635$ $\pm 5 \mathrm{kA} / \mathrm{m}$, lower than the value of $M_{s}=938 \pm 1 \mathrm{kA} / \mathrm{m}$ obtained for the undoped film. The reduction in $\mathrm{M}_{\mathrm{s}}$ for Tb-doped films is well-known and occurs due to the antiferromagnetic coupling of $\mathrm{Tb}$ moments to the ferromagnetically coupled $\mathrm{Ni}$ and Fe moments ${ }^{45,46}$. We note that the values of $\alpha$ and $\mathrm{M}_{\mathrm{s}}$ for the undoped films were respectively lower and higher than would be expected for stoichiometric $\mathrm{Ni}_{80} \mathrm{Fe}_{20}$ films, perhaps suggesting that they were slightly iron rich. 


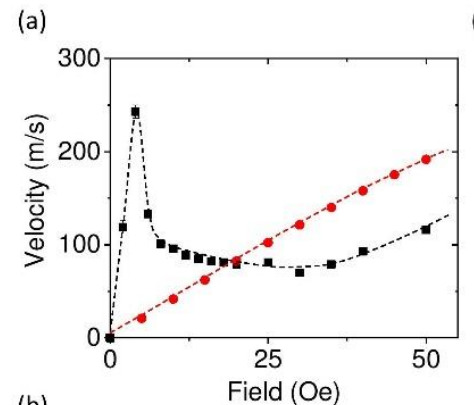

(c)
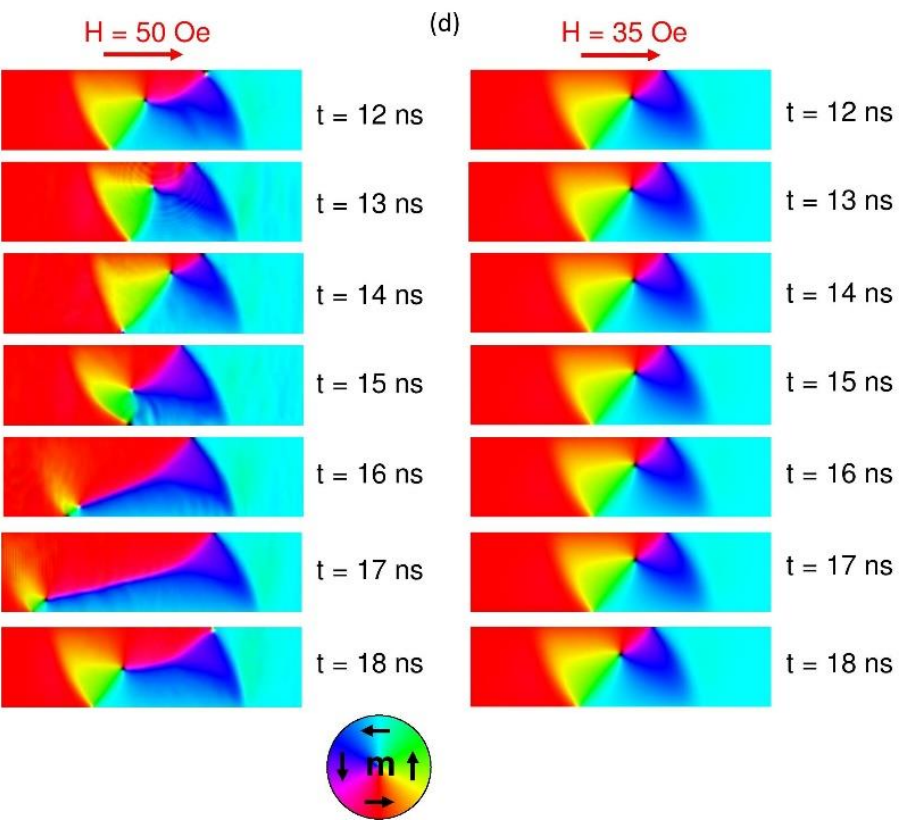

Figure 3: (a) Micromagnetically calculated domain wall velocities versus applied field in $\mathrm{Ni}_{80} \mathrm{Fe}_{20}$ (black squares) and $5 \% \mathrm{~Tb}$-doped $\mathrm{Ni}_{80} \mathrm{Fe}_{20}$ nanowires (red circles). (b) Simulated plots of domain wall position versus time at the average injection fields of the undoped and $5 \%$ Tb-doped nanowires. The plots have been distributed vertically to improve visibility. (c) Simulated domain wall dynamics at $H=50$ Oe in an undoped nanowire. The mesh window was dynamically centred on the domain wall in each iteration of the simulation. (d) Simulated DW dynamics at $H=35$ Oe in a Tb-doped nanowire.

To estimate the degree to which the enhanced damping of the Tb-doped films would suppress Walker breakdown dynamics we performed micromagnetic simulations of DW propagation in nanowires matching the experimental geometry. Figure 3(a) presents the variation of simulated DW velocity with applied field for nanowires with material properties matching those of both the Tb-doped and undoped films. The data for the undoped nanowire followed the established form for DWs in soft ferromagnetic nanowires: ${ }^{33,38}$ Initially, the velocity of the DW increased linearly with applied field, with this trend terminating at $\mathrm{H}_{\mathrm{WB}}=4 \mathrm{Oe}$. After this point DW motion entered the oscillatory regime, where the DW structure underwent periodic cycles of transformation, resulting in a dramatic reduction in velocity. The velocity of the DW then remained constant until approximately $\mathrm{H}=40 \mathrm{Oe}$, at which point it started to increase again due to the DW entering the turbulent regime of motion, where the periodicity of the DW transformations broke down. In contrast to this, the plot for the Tb-doped nanowires showed a simple monotonic trend, indicating that $\mathrm{H}_{\mathrm{WB}}$ had been increased to a point beyond the plotted field range. Our simulations suggested that $\mathrm{H}_{\mathrm{WB}}>100$ Oe for the doped nanowires.

Figures 3(b) - (d) present DW dynamics for two particular simulations: propagation at $\mathrm{H}=50 \mathrm{Oe}$ in the undoped nanowire and at $\mathrm{H}=35 \mathrm{Oe}$ in the Tb-doped nanowire. We selected these two fields because, as we show below, they represent the mean DW injection fields for nanowires fabricated from the 
precusor films. Figure 3(b) illustrates the time-dependence of the DWs' positions under these conditions. The data for the undoped nanowire shows quasi-periodic oscillations, suggesting that the mean injection field for these devices lay just above the boundary between the oscillatory and turbulent regimes of motion. This is consistent with the DW dynamics shown in Figure 3(c), where the DW was continuously transforming between VDW-like and transverse DW-like (TDW-like) configurations. In contrast to this, the position of the DW in the Tb-doped nanowire increased monotonically with time (Figure 3(d)), and the DW retained a consistent VDW magnetisation structure, confirming that the mean injection field in these devices lay in the viscous regime of DW motion, below $\mathrm{H}_{\text {พв. }}$. While exact quantitative agreement between our micromagnetic simulations and the DW dynamics in the devices was not expected, the large differences between the injection fields and Walker breakdown fields in both sets of simulations allowed us to have confidence that DWs would propagate with stable magnetisation structures in the doped nanowires and unstable structures in the undoped nanowires. Nanowires fabricated from the Tb-doped films were thus expected to show substantially more deterministic behaviour than equivalents fabricated from the undoped films. We also note that, despite DW mobility in the viscous regime typically decreasing with increasing $\alpha$, DWs in the doped nanowires were predicted to propagate slightly faster than those in the undoped nanowires at their typical injection fields, due to the suppression of Walker breakdown in the doped devices.

Having demonstrated that $H_{W B}$ should be substantially increased in nanowires with the material properties of the Tb-doped film, we fabricated nanowire devices similar to those shown in Figure 1(a) from both the Tb-doped and undoped films, and characterised their IFDs and DFDs using FMOKE measurements. For the Tb-doped nanowires (the primary focus of the study) eight devices were measured per defect geometry, with the final data set restricted to devices that exhibited (a) typical injection fields ( $H_{\text {injec }}<50 \mathrm{Oe}$ ) and (b) an absence of additional domain wall pinning sites between the pad and the lithographically patterned notch defect. Additional pinning sites were easily identified as steps in the nanowires' hysteresis loops at FMOKE laser spot positions between the nucleation pad and the notch. We restricted our final data set in this way to ensure that our understanding of the dynamical interactions of the DWs with the notch defects was not distorted by (a) DWs in some devices interacting with the notches at anomalously high fields or (b) by additional defect sites introducing chirality filtering effects (and thus additional stochasticity) prior to the DWs' interactions with the notches. Applying these criteria restricted our data set to between 4 and 6 devices per notch geometry. For the undoped nanowires, which acted as a reference data set, four nanowires were measured per defect geometry. All these devices were found to meet the criteria above and contributed to our final dataset. We suspect the difference in the viability of devices between the 
doped and undoped nanowires was due to the enhanced reliability of pinning in the Tb-doped devices (see below), which meant that even small edge defects could pin DWs. Similar defects were likely passed through dynamically in the undoped nanowires. ${ }^{17}$

Figures 4(a)-(d) present representative examples of measurements for nanowires containing defects with a variety of sizes $\left(d_{n}=40-165 n m\right.$ and $\left.w_{n}=130-375 n m\right)$. A more comprehensive set of results, comprising measurements from a minimum of four devices for each defect geometry/material combination can be found in the supplementary material ${ }^{49}$

The data from the undoped nanowires, for all defect sizes, exhibited highly stochastic DW pinning/depinning. Each nanowire exhibited multi-mode DFDs, indicating that DWs with different magnetisation structures were pinning at the defect sites, while nanowires with smaller notches exhibited substantial overlap between their IFDs and DFDs, indicating that pinning was probabilistic. Thus, the data showed all the key features of dynamically induced stochastic DW behaviour as one would expect when DWs are incident upon a defect site at fields above $H_{w B}$.

In striking contrast to this, the devices fabricated from the Tb-doped films showed quasi-deterministic behaviour. Most notably, for all defect sizes DFDs and IFDs were well-separated, indicating that DWs pinned $100 \%$ reliably at the defect (i.e. a complete suppression of stochastic pinning effects). Furthermore, the majority of devices (11/20) exhibited sharp, single mode DFDs, indicating that DWs pinned with consistent, magnetisation structure. The remaining devices shown exhibited more than one mode (see for example Figure $4(d)$ ), but these remained relatively tightly distributed (typically within $\sim 30$ Oe), such that for a given defect geometry the DFD was notably narrower in the Tb-doped nanowires (i.e. a significant suppression of stochastic depinning). We will return to the question of why these devices showed residual stochasticity in their DFDs, despite DWs propagating to the defect sites at fields significantly below $\mathrm{H}_{\text {WB }}$ shortly. 

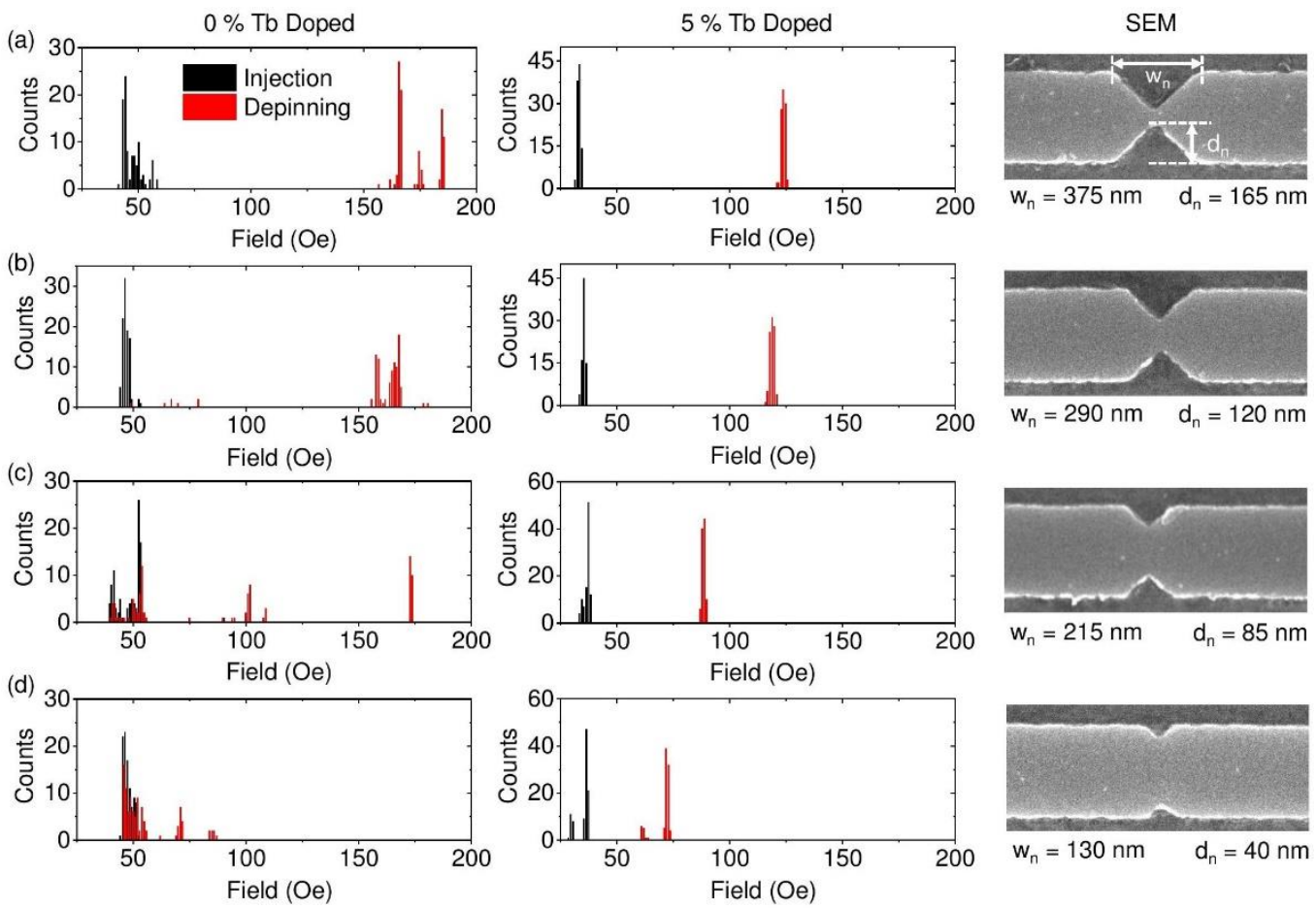

Figure 4: IFDs and DFDs measured from undoped $\mathrm{Ni}_{80} \mathrm{Fe}_{20}$ and $5 \% \mathrm{~Tb}$-doped nanowires for a variety of artificial defect sizes. The scanning electron microscope (SEM) images at the right hand side of the figure show representative defect shapes and dimensions for each geometry studied.

The contrasting behaviour of the two sets of devices is illustrated further in Figures 5(a)-(c), which present mean values of the DFDs $\left(\bar{H}_{d}\right)$, pinning probabilities and DFD standard deviations $\left(\bar{\sigma}_{\text {depin }}\right)$ as a function of $d_{n}$. In all three plots, each data point represents an average of the parameter in question taken across the measured devices with identical notch depths $d_{n}$ and material compositions. Error bars in all three figures represent the standard deviation of the data set contributing to that point, rather than the standard error, in order to give the reader a clear indication of the spread of values across the devices measured. 

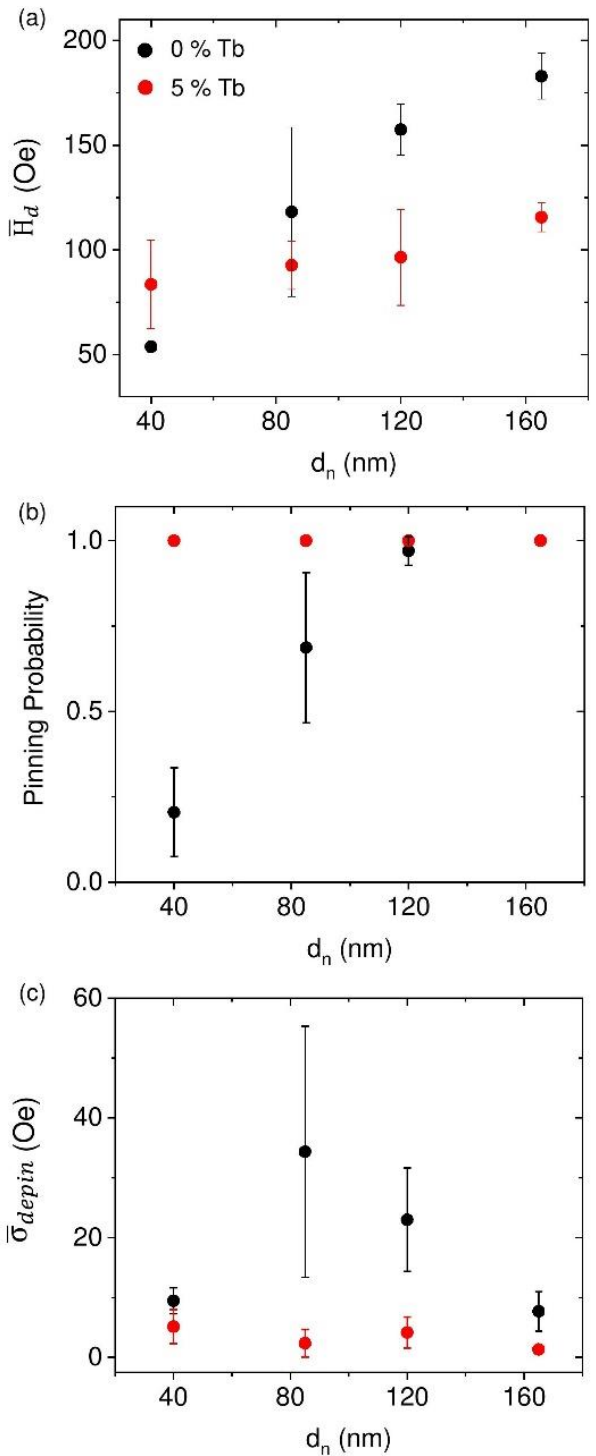

Figure 5: (a) Mean depinning field $\left(\bar{H}_{d}\right)$ as a function of notch depth $\left(d_{n}\right)$ for the undoped $\mathrm{Ni}_{80} \mathrm{Fe}_{20}$ (black circles) and $5 \%$ Tb-doped (red circles) nanowires. (b) Mean pinning probability as a function of notch depth. (c) Mean DFD standard deviation ( $\left.\bar{\sigma}_{\text {depin }}\right)$, as a function of notch depth. In all three plots each data point represents an average of the parameter in question taken across measured devices with nominally identical notch depths $d_{n}$ and material compositions. Error bars in all three figures represent the standard deviation of the data set contributing to the point, rather than the standard error, to give the reader a clear indication of the spread of values across the devices measured.

Figure 5(a) shows that, for both the Tb-doped and undoped nanowires, $\bar{H}_{d}$, increased monotonically with $d_{n}$, as would be expected from the increased pinning potentials that larger defects presented. We note that the variation was substantially greater for the undoped nanowires, a feature that we attribute to the fractional pinning probabilities of these nanowires, which biased the means of the DFDs towards lower values for lower values of $d_{n}$. 
The dependence of the pinning probabilities on $d_{n}$ are presented in figure $5(b)$ where it can be seen that the undoped nanowires showed highly stochastic pinning for $d_{n}<100 \mathrm{~nm}$, replicating trends seen in several of our previous studies. ${ }^{4,39}$ In contrast to this, the Tb-doped nanowires exhibited $100 \%$ reliable pinning for each of the defect geometries studied.

A more generalised expression of the stochasticity is provided by $\bar{\sigma}_{\text {depin }}$ (Figure $5(c)$ ), which we consider here as a crude measure of DFD spread. For the Tb-doped nanowires $\bar{\sigma}_{\text {depin }}$ was typically just a few Oersted, emphasising the highly repeatable switching in these devices. The values of $\bar{\sigma}_{\text {depin }}$ for the undoped nanowires were universally higher than this, peaking at $\bar{\sigma}_{\text {depin }}=34$ Oe for $d_{n}=85 \mathrm{~nm}$, an enhancement of $\sim 1400 \%$ over the value $\bar{\sigma}_{\text {depin }}=2.4$ Oe observed for equivalent Tb-doped nanowires. We note that the relatively low value of $\bar{\sigma}_{\text {depin }}=9.5$ Oe observed for the smallest defects $\left(d_{n}=40 \mathrm{~nm}\right)$ in the undoped nanowires was purely a consequence of the low pinning probability (i.e. the DFD overlapped strongly with the relatively narrow IFD), and thus was somewhat misleading. On the other hand, the low value $\sigma_{\text {depin }}=7.7$ Oe for the largest defect sites $\left(d_{n}=165 n m\right)$ in the undoped nanowires genuinely reflected the tendency for dynamically induced stochastic effects to be less significant for larger defect sites, ${ }^{4,39}$ and yet was still $600 \%$ larger than the value for equivalently sized defects in the doped nanowires. These trends were maintained even when we normalised $\sigma_{\text {depin }}$ to $H_{\text {depin }}$ to take account of generally higher depinning fields in the undoped nanowires (see the supplementary material ${ }^{49}$ ).

We note here that, while previous work has shown that even in undoped nanowires rare defect geometries can be found that minimise stochasticity ${ }^{4,39}$, we have observed substantial improvements in both metrics of stochasticity (pinning probability and $\bar{\sigma}_{\text {depin }}$ ) across all defect geometries in the doped nanowires. This suggests these improvements originate in the material properties of the nanowires, rather than an accident of device geometry.

While the results presented above clearly demonstrate that stochastic behaviour was dramatically suppressed in the Tb-doped nanowires, we now return to the origin of the small degree of DFD splitting observed in a minority of the these devices This is unexpected, as suppression of Walker breakdown should have resulted in the DWs adopting singular, well-defined magnetisation configurations when pinned at defect sites and thus single mode DFDs. In several nanowires (e.g. Figure 4(d)) splitting in both the IFD and DFD appeared simultaneously, prompting us to investigate whether there was a causal relationship between bimodal/split IFDs and split DFDs, and a Pearson's $\chi^{2}$ test $\left(\chi^{2}=5.09\right.$ and $\left.P=0.024\right)$ rejected the null hypothesis that these features were uncorrelated across the data set at $P<0.05$ (see supplementary material ${ }^{49}$ ). 
To explain the correlation between splitting in the IFDs and DFDs we further studied the nanowires behaviours using micromagnetic simulations. We first note that these indicated that the total energy of TDWs were only $30 \%$ greater than that of VDWs in the doped nanowires, and that both types of DWs could propagate stably at typical injection fields. Thus, the doped nanowires could feasibly support six distinct propagating DW structures: TDWs with UP/DOWN chiralities, and VDWs with $\mathrm{ACW} / \mathrm{CW}$ chiralities and up/down vortex core polarisations. We suggest that in devices where the IFDs were split (i.e. exhibited multiple reversal modes), multiple forms of DWs were being injected into the nanowires. The different ways in which these pinned at a given defect site would then result in subsequent splitting of the DFD. We note that even in devices with single mode IFDs it is possible that different DW structures could be injected, for example through the effects of thermal perturbations at critical points in the injection process. Furthermore, different injection modes might not always result in different DW structures being injected. Potentially, this explains why splitting of the IFDs and DFDs were not perfectly correlated across our data set.

To test this hypothesis we used micromagnetic simulations to examine the pinning and depinning of each possible DW structure at a defect site replicating the geometry of the double notches shown in Figure $4(d)\left(d_{n}=40 \mathrm{~nm}\right)$. Details of these simulations are presented in the supplementary material ${ }^{49}$, Figure S6. The simulations showed that DWs could exhibit three distinct depinning fields: $\mathrm{H}=60 \mathrm{Oe}$ (ACW VDW with core up/down), H $=75$ Oe (CW VDW with core down) and 85 Oe (TDW UP, TDW DOWN, CW VDW with core up). The range of these depinning fields corresponds closely to that observed for experimental devices with similar notch geometries (Figure S4). Thus, we conclude the small degree of DFD stochasticity observed in a minority of the Tb-doped devices was likely due to variability in the magnetisation structure of injected DWs, as opposed to inherently stochastic interaction of the DWs with the defect sites. This is an important distinction, as many studies have demonstrated the feasibility of controlling the initial structure/chirality of $D W s^{6,24,50,51}$, while the dynamic interactions of DWs with defect sites is inherently uncontrollable at fields above $\mathrm{H}_{\mathrm{WB}}{ }^{38}$. Clearly, methods of controlling the structure of nucleated DWs will need be employed in future devices to obtain fully reliable operations.

DWs in soft magnetic nanowires are known to strongly manifest dynamically induced stochasticity, and thus they were expected to benefit dramatically from the materials engineering approach we have demonstrated here. However, it is interesting to ask whether the approach might have broader utility. To examine this, we considered the $10 \times 10 \mu \mathrm{m}^{2}$ nucleation pads attached to the nanowires' ends. These represented more generic examples of mesoscopic magnetic systems, and were expected to exhibit complex 2-dimensional magnetisation states, ${ }^{52}$ the details of which would dictate the manner in which DWs were injected into the nanowires. Thus, the widths of IFDs were expected to 
offer a probe of how stochastic the switching behaviours of these large, complex magnetic microstructures were.

A cursory examination of the IFDs shown in Figure 4 appeared to indicate that the IFDs for the undoped nanowires were typically wider and more complex than those for the Tb-doped nanowires. Quantitatively, we found that the mean of the IFD standard deviations was, $\sigma_{\text {inject }}=3.8 \pm 0.6$ Oe for the undoped nanowires compared to $\sigma_{\text {inject }}=1.6 \pm 0.3$ Oe for the Tb-doped nanowires. This striking reduction in stochasticity remained significant even when these results were normalised by the nanowires' mean injection fields (undoped nanowires: $\sigma_{\text {inject }} / H_{\text {inject }}=0.078 \pm 0.012$, Tb-doped nanowires: $\sigma_{\text {inject }} / H_{\text {inject }}=0.045 \pm 0.010$ ). These additional results thus evidence that engineering the dynamical behaviours of magnetic materials to reduce stochasticity can find utility in a wider range of systems than those we have primarily studied here.

\section{Conclusions}

In this paper we have demonstrated that materials engineering can be used to control dynamically induced stochastic behaviour in magnetic nanostructures. Using the pinning of DWs in $\mathrm{Ni}_{80} \mathrm{Fe}_{20}$ nanowires as a case study, we have shown how enhancing the damping constant of magnetic materials via doping with rare earth elements allows us to obtain simple, quasi-deterministic switching behaviour from systems that nominally show exceptionally complex stochastic behaviours. Our work demonstrates that engineering basic magnetic properties offers a viable route to regulating device behaviour, even when stochasticity is caused by the inherent spatial and temporal complexity of magnetisation dynamics, rather than by simple thermal activation processes.

More directly, our results have potential implications for devices based on the propagation and pinning of DWs in soft ferromagnetic nanowires. For example, we have recently proposed DW logic networks in which binary information is stored and processed within the chirality of VDWs ${ }^{21,24}$ and the viability of such architectures will be entirely dependent on stabilising the DW structure and obtaining reliable DW pinning/depinning. Furthermore, automotive turn sensors based on soft magnetic nanowires are currently the only commercial realisation of DW devices ${ }^{28,29}$, and their noise performance may be improved by the suppression of stochastic pinning effects. Architecturally similar devices for transporting magnetic micro/nano-particles ${ }^{22,30}$ and ultra-cold atoms ${ }^{25,53,54}$ may also benefit from smoother, more deterministic DW motion. In real optimised doping levels would need to be selected. These would be chosen to keep DWs moving at fields just below Walker breakdown for typical operating fields/currents (thus minimising stochasticity), while also maximising the DW mobility and thus the operating speeds of devices. 
Our work will also have interesting implications for artificial spin ice systems, the dynamics of which are intimately linked to the types of DW dynamics studied here ${ }^{5,6}$. These have proposed applications in the rapidly emerging field of neuromorphic computing 55,56 , and the ability to tune the level of stochasticity in such systems may be highly valuable to realising viable devices.

\section{Acknowledgements}

T.J.H acknowledges support by the Engineering and Physical Sciences Research Council (Grant No:

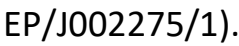




\section{References}

1. G. W. Qin et al. Development of high density magnetic recording media for hard disk drives: Materials science issues and challenges. Int. Mater. Rev. 54, 157-179 (2009).

2. A. D. Kent \& D. C. Worledge. A new spin on magnetic memories. Nature Nanotechnology vol. 10 187-191 (2015).

3. W. F. Brown. Thermal fluctuations of a single-domain particle. Phys. Rev. 130, 1677-1686 (1963).

4. T. J. Hayward \& K. A. Omari. Beyond the quasi-particle: Stochastic domain wall dynamics in soft ferromagnetic nanowires. J. Phys. D. Appl. Phys. 50, 084006 (2017).

5. K. Zeissler et al. The non-random walk of chiral magnetic charge carriers in artificial spin ice. Sci. Rep. 3, 1252 (2013).

6. A. Pushp et al. Domain wall trajectory determined by its fractional topological edge defects. Nat. Phys. 9, 505-511 (2013).

7. M. Y. Im et al. Stochastic formation of magnetic vortex structures in asymmetric disks triggered by chaotic dynamics. Nat. Commun. 5, 5620 (2014).

8. A. M. Shutyi \& D. I. Sementsov. Magnetization Stochastic Dynamics in Exchange-Coupled Layered Structureo Title. JEPT Lett. 78, 480-484 (2003).

9. D. I. Sementsov \& A. M. Shutyi. Nonlinear regular and stochastic dynamics of magnetization in thin-film structures. Phys.-Usp 50, 793 (2007).

10. E. Reibold, W. Just, J. Becker \& H. Benner. Stochastic Resonance in Chaotic Spin-Wave Dynamics. Phys. Rev. Lett 78, 1997 (1997).

11. M. Hayashi et al. Dependence of current and field driven depinning of domain walls on their structure and chirality in permalloy nanowires. Phys. Rev. Lett. 97, 207205 (2006).

12. H. Tanigawa et al. Dynamical pinning of a domain wall in a magnetic nanowire induced by walker breakdown. Phys. Rev. Lett. 101, 207203 (2008).

13. M. Y. Im, L. Bocklage, P. Fischer \& G. Meier. Direct observation of stochastic domain-wall depinning in magnetic nanowires. Phys. Rev. Lett. 102, 147204 (2009).

14. M. Muñoz \& J. L. Prieto. Suppression of the intrinsic stochastic pinning of domain walls in magnetic nanostripes. Nat. Commun. 2, 562 (2011). 
15. U. H. Pi et al. Static and dynamic depinning processes of a magnetic domain wall from a pinning potential. Phys. Rev. B - Condens. Matter Mater. Phys. 84, 024426 (2011).

16. J. Briones et al. Stochastic and complex depinning dynamics of magnetic domain walls. Phys. Rev. B - Condens. Matter Mater. Phys. 83, 060401(R) (2011).

17. E. R. Lewis et al. Kinetic depinning of a magnetic domain wall above the Walker field. Appl. Phys. Lett. 98, 042502 (2011).

18. S. S. P. Parkin, M. Hayashi \& L. Thomas. Magnetic domain-wall racetrack memory. Science vol. 320 190-194 (2008).

19. S. Parkin \& S.-H. Yang. Memory on the racetrack. Nature Nanotechnology vol. 10 195-198 (2015).

20. D. A. Allwood et al. Magnetic domain-wall logic. Science vol. 309 1688-1692 (2005).

21. K. A. Omari \& T. J. Hayward. Chirality-Based Vortex Domain-Wall Logic Gates. Phys. Rev. Appl. 2, 044001 (2014).

22. E. Rapoport, D. Montana \& G. S. D. Beach. Integrated capture, transport, and magnetomechanical resonant sensing of superparamagnetic microbeads using magnetic domain walls. Lab Chip 12, 4433-4440 (2012).

23. J. Vandermeulen, B. Van De Wiele, L. Dupré \& B. Van Waeyenberge. Logic and memory concepts for all-magnetic computing based on transverse domain walls. J. Phys. D. Appl. Phys. 48, 275003 (2015).

24. K. A. Omari et al. Toward Chirality-Encoded Domain Wall Logic. Adv. Funct. Mater. 29, 1807282 (2019).

25. D. A. Allwood, T. Schrefl, G. Hrkac, I. G. Hughes \& C. S. Adams. Mobile atom traps using magnetic nanowires. Appl. Phys. Lett. 89, 014102 (2006).

26. T. J. Hayward et al. Nanomagnetic engineering of the properties of domain wall atom traps. J. Appl. Phys. 110, 123918 (2011).

27. A. D. West et al. Realization of the manipulation of ultracold atoms with a reconfigurable nanomagnetic system of domain walls. Nano Lett. 12, 4065-4069 (2012).

28. R. Mattheis, M. Diegel, U. Hübner \& E. Halder. Multiturn counter using the movement and storage of $180^{\circ}$ magnetic domain walls. IEEE Trans. Magn. 42, 3297-3299 (2006). 
29. R. Mattheis, S. Glathe, M. Diegel \& U. Hübner. Concepts and steps for the realization of a new domain wall based giant magnetoresistance nanowire device: From the available 24 multiturn counter to a 212 turn counter. in Journal of Applied Physics vol. 111113920 (2012).

30. E. Rapoport \& G. S. D. Beach. Architecture for Directed Transport of Superparamagnetic Microbeads in a Magnetic Domain Wall Routing Network. Sci. Rep. 7, 10139 (2017).

31. S. Ladak, D. E. Read, G. K. Perkins, L. F. Cohen \& W. R. Branford. Direct observation of magnetic monopole defects in an artificial spin-ice system. Nat. Phys. 6, 359-363 (2010).

32. W. R. Branford, S. Ladak, D. E. Read, K. Zeissler \& L. F. Cohen. Emerging chirality in artificial spin ice. Science (80-. ). 335, 1597-1600 (2012).

33. G. S. D. Beach, C. Nistor, C. Knutson, M. Tsoi \& J. L. Erskine. Dynamics of field-driven domainwall propagation in ferromagnetic nanowires. Nat. Mater. 4, 741-744 (2005).

34. N. Vernier, D. A. Allwood, D. Atkinson, M. D. Cooke \& R. P. Cowburn. Domain wall propagation in magnetic nanowires by spin-polarized current injection. Europhys. Lett. 65, 526-532 (2004).

35. N. L. Schryer \& L. R. Walker. The motion of $180^{\circ}$ domain walls in uniform dc magnetic fields. J. Appl. Phys. 45, 5406-5421 (1974).

36. J. Y. Lee, K. S. Lee, S. Choi, K. Y. Guslienko \& S. K. Kim. Dynamic transformations of the internal structure of a moving domain wall in magnetic nanostripes. Phys. Rev. B - Condens. Matter Mater. Phys. 76, 184408 (2007).

37. S. K. Kim, J. Y. Lee, Y. S. Choi, K. Y. Guslienko \& K. S. Lee. Underlying mechanism of domainwall motions in soft magnetic thin-film nanostripes beyond the velocity-breakdown regime. Appl. Phys. Lett. 93, 052503 (2008).

38. T. J. Hayward. Intrinsic Nature of Stochastic Domain Wall Pinning Phenomena in Magnetic Nanowire Devices. Sci. Rep. 5, 13279 (2015).

39. K. A. Omari \& T. J. Hayward. A Micromagnetic Protocol for Qualitatively Predicting Stochastic Domain Wall Pinning. Sci. Rep. 7, 17862 (2017).

40. S. S. Kalarickal et al. Ferromagnetic resonance linewidth in metallic thin films: Comparison of measurement methods. J. Appl. Phys. 99, 093909 (2006).

41. R. D. McMichael \& M. J. Donahue. Head to head domain wall structures in thin magnetic strips. IEEE Trans. Magn. 33, 4167-4169 (1997). 
42. M. Laufenberg et al. Observation of thermally activated domain wall transformations. Appl. Phys. Lett. 88, 1-3 (2006).

43. T. J. Broomhall \& T. J. Hayward. Suppression of Stochastic Domain Wall Pinning Through Control of Gilbert Damping. Sci. Rep. 7, 17100 (2017).

44. A. Vansteenkiste et al. The design and verification of MuMax3. AIP Adv. 4, 107133 (2014).

45. W. Bailey, P. Kabos, F. Mancoff \& S. Russek. Control of magnetization dynamics in Ni81Fe19 thin films through the use of rare-earth dopants. IEEE Trans. Magn. 37, 1749-1754 (2001).

46. G. Woltersdorf, M. Kiessling, G. Meyer, J. U. Thiele \& C. H. Back. Damping by slow relaxing rare earth impurities in Ni80Fe20. Phys. Rev. Lett. 102, 257602 (2009).

47. T. A. Moore et al. Magnetic-field-induced domain-wall motion in permalloy nanowires with modified Gilbert damping. Phys. Rev. B - Condens. Matter Mater. Phys. 82, 094445 (2010).

48. D. G. Porter \& M. J. Donahue. Velocity of transverse domain wall motion along thin, narrow strips. in Journal of Applied Physics vol. 95 6729-6731 (2004).

49. See Supplemental Material at [URL will be inserted by publisher] for histogram/numerical data from each devices measured, details of statisical testing and additional simulation results.

50. D. Mcgrouther, S. McVitie, J. N. Chapman \& A. Gentils. Controlled domain wall injection into ferromagnetic nanowires from an optimized pad geometry. Appl. Phys. Lett. 91, 022506 (2007).

51. K. Omari et al. Ballistic rectification of vortex domain wall chirality at nanowire corners. Appl. Phys. Lett. 107, (2015).

52. M. T. Bryan et al. Transverse field-induced nucleation pad switching modes during domain wall injection. IEEE Trans. Magn. 46, 963-967 (2010).

53. M. Negoita, T. J. Hayward \& D. A. Allwood. Controlling domain walls velocities in ferromagnetic ring-shaped nanowires. Appl. Phys. Lett. 100, 072405 (2012).

54. M. Negoita, T. J. Hayward, J. A. Miller \& D. A. Allwood. Domain walls in ring-shaped nanowires under rotating applied fields. J. Appl. Phys. 114, 013904 (2013).

55. M. Hehn et al. Bio-Inspired computing based on artificial spin ices (Conference Presentation). in Spintronics XI 121 (2018). doi:10.1117/12.2325724. 
56. J. H. Jensen, E. Folven \& G. Tufte. Computation in artificial spin ice. in ALIFE 2018: The 2018 Conference on Artificial Life 15-22. 